\title{
Color-spin locking in a self-consistent Dyson-Schwinger approach
}

\author{
Florian Marhauser, ${ }^{1}$ Dominik Nickel, ${ }^{1}$ Michael Buballa, ${ }^{1}$ and Jochen Wambach ${ }^{1,2}$ \\ ${ }^{1}$ Institut für Kernphysik, Technische Universität Darmstadt, D-64289 Darmstadt, Germany \\ ${ }^{2}$ Gesellschaft für Schwerionenforschung mbH, Planckstraße 1, D-64291 Darmstadt, Germany
}

(Dated: October 9, 2018)

\begin{abstract}
We investigate the color-spin locked (CSL) phase of spin-one color-superconducting quark matter using a truncated Dyson-Schwinger equation for the quark propagator in Landau gauge. Starting from the most general parity conserving ansatz allowed by the CSL symmetry, the Dyson-Schwinger equation is solved self-consistently and dispersion relations are discussed. We find that chiral symmetry is spontaneously broken due to terms which have previously been neglected. As a consequence, the excitation spectrum contains only gapped modes even for massless quarks. Moreover, at moderate chemical potentials the quasiparticle pairing gaps are several times larger than expected from extrapolated weak-coupling results.
\end{abstract}

\section{INTRODUCTION}

In recent years, the possible existence of various color superconducting phases in Quantum Chromo Dynamics (QCD) at low temperatures and sufficiently high densities has received much attention (for reviews see [1, 2]). At asymptotic densities, where the problem can be treated within a weak-coupling expansion, there is compelling evidence [3] that quark matter is in the colorflavor locked (CFL) phase where up, down, and strange quarks participate in a scalar (spin 0) diquark condensate [4]. The situation is much less clear at "moderate" densities of about 3 to 10 times nuclear matter density, which could be reached in the centers of compact stars. These densities could be too small to allow for a sizeable fraction of strange quarks and, hence, pairing of strange quarks with nonstrange quarks might be disfavored. On the other hand, the cross-flavor pairing of up and down quarks in a two-flavor superconducting (2SC) phase could be unfavorable as well if local neutrality and beta equilibrium are taken into account [5]. Indeed, as demonstrated in various model calculations, BCS pairing of quarks with unequal flavors could be absent in the relevant regions of the phase diagram [6, 7].

In such environments quarks of different flavors might pair independently. In this case, if we restrict ourselves to pairing channels which are antisymmetric in color, spin-0 pairing is Pauli-forbidden and the most promising candidates are Cooper pairs with total spin 1. Such phases were discussed first in Refs. [8, 9] and their properties were investigated in great detail in Ref. [10] for weakcoupling. It was found that, among the various pairing patterns, the most stable spin- 1 phase is the so-called color-spin locked (CSL) phase, where the three spin projections are locked to the three color degrees of freedom of the antitriplet. This is quite analogous to the $\mathrm{B}$ phase of superfluid helium-3, where the spin is locked to the orbital angular momentum.

In Ref. 11] the CSL phase has been analyzed within an Nambu-Jona-Lasinio (NJL)-type model. In this model, the locking of color and spin was introduced through a gap matrix

$$
\Phi^{+}=\Delta \vec{\gamma} \cdot \vec{I}
$$

where $\vec{\gamma}=\left(\gamma_{1}, \gamma_{2}, \gamma_{3}\right)$ are the gamma matrices with spacelike indices and $\vec{I}=\left(\lambda_{7},-\lambda_{5}, \lambda_{2}\right)$ are the antisymmetric Gell-Mann matrices, acting in color space. It was found that for massive quarks all quasiparticle modes are gapped with the smallest gap in the spectrum being proportional to the constituent quark mass. This is an interesting result because ungapped quark matter in general leads to rapid neutron star cooling via the direct URCA process which is difficult to reconcile with the empirical data. In fact, in a recent analysis of neutron star cooling data, it was concluded that in a possible quark core all quasiparticle modes should be gapped with the smallest gap of the order of 10-100 keV and decreasing with density [12]. The NJL-model results of Ref. [11] turned out to be in rough agreement with these constraints. However, the size of the smallest gap was found to be very sensitive to the model parameters (see also Ref. 13], where a similar model with a nonlocal interaction was used). Moreover, in order to obtain gaps which are decreasing with density the authors used a density dependent coupling which had to be introduced by hand. In this situation a more fundamental, QCD-based approach is clearly desirable.

A second problem was raised in Ref. [14]. Here the color-spin locking was introduced in a similar way as in Eq. (11) but with $\vec{\gamma}$ replaced by a transverse gamma matrix, $\vec{\gamma}_{\perp}(\vec{p})=\vec{\gamma}-(\vec{\gamma} \cdot \hat{p}) \hat{p}$, where $\hat{p}=\vec{p} /|\vec{p}|$ and $\vec{p}$ is the 3 -momentum dependence of the gap matrix. In this case it turns out that there is always an ungapped mode, even for massive quarks. Of course, the question whether or not there are ungapped modes in the spectrum should not depend on an arbitrary ansatz for the gap matrix. Instead, the gap structure should be the result of a selfconsistent dynamical calculation searching for the most favored ground state. Again, this problem cannot be solved within NJL-type models, because the point interaction does not allow for momentum dependent structures, like $\vec{\gamma}_{\perp}$.

Recently, there has been considerable progress in 
studying QCD at nonzero chemical potential within the framework of Dyson-Schwinger equations (DSEs) in Landau gauge [15, 16, 17]. Dyson-Schwinger equations provide a set of coupled integral equations that are the quantum equations of motion of the Green functions of a quantum field theory. If they are solved completely, the whole dynamics of the theory under consideration is understood. Of course, in practice, one has to perform truncations. By now there is a lot of experience in setting up a truncation scheme for Landau gauge in the vacuum, whose results are in good agreement with lattice data (for reviews see [18]). In Refs. [15, 17] this scheme has been extended to describe spin- 0 color superconductors at nonzero chemical potential. This has been done in such a way that at very large chemical potentials the weak-coupling results of Ref. [19] are correctly reproduced. To that end, medium modifications of the gluon propagator through particle-hole excitations have been implemented.

Thus being constrained from "two ends" (lattice calculations in vacuum and weak-coupling results at very large $\mu$ ), the framework has then been applied to the "interesting" regime of moderate chemical potentials. Here the authors found considerable deviations from both the naively extrapolated weak-coupling pairing gaps [15] and the NJL-model behavior of the dynamical quark masses [17.

Motivated by these results, we have extended the formalism of Refs. 15, 17] to describe spin-1 color superconductors in the CSL phase. Main focus is thereby the question about the smallest excitation gap, raised by the (partially contradicting) results of Refs. [11] and 14]. To avoid similar problems related to a specific ansatz for the gap function, we set up a fully self-consistent scheme, starting from the most general parity conserving CSL symmetric ansatz for the normal and anomalous quark self-energies. Since in Ref. [11] (constituent) quark masses were found to be important, we also include chiral symmetry breaking terms. As we will see, these terms are in fact crucial and, to our surprise, even survive in the chiral limit.

This work is organized in the following way: In section III we give a brief presentation of the theoretical framework for our self-consistent solution of the DysonSchwinger equation. The numerical results are presented in section [III. In section IV we present a physical interpretation in terms of dispersion relations. We summarize what we accomplished and conclude in section $\mathrm{V}$.

\section{THEORETICAL SETUP}

\section{A. The truncated quark DSE at finite chemical potential}

We follow the scheme and notations presented in [15] to get a truncated, but closed DSE for the quark propagator at a finite chemical potential within the Nambu-
Gor'kov formalism. The renormalized quark DSE in Landau gauge with appropriate quark-wave function and quark-gluon vertex renormalization constants, $Z_{2}$ and $Z_{1 F}$, respectively, is then given by

$$
\mathcal{S}^{-1}(p)=Z_{2} \mathcal{S}_{0}^{-1}(p)+Z_{1 F} \Sigma(p) .
$$

$\mathcal{S}_{0}$ denotes the bare quark propagator, $\mathcal{S}$ the full quark propagator and $\Sigma$ the quark self-energy. These objects are $2 \times 2$ matrices in Nambu-Gor'kov space, consisting of normal and anomalous components:

$$
\begin{aligned}
\mathcal{S}_{0}(p) & =\left(\begin{array}{cc}
S_{0}^{+}(p) & 0 \\
0 & S_{0}^{-}(p)
\end{array}\right), \\
\mathcal{S}(p) & =\left(\begin{array}{cc}
S^{+}(p) & T^{-}(p) \\
T^{+}(p) & S^{-}(p)
\end{array}\right), \\
\Sigma(p) & =\left(\begin{array}{cc}
\Sigma^{+}(p) & \Phi^{-}(p) \\
\Phi^{+}(p) & \Sigma^{-}(p)
\end{array}\right) .
\end{aligned}
$$

Inserting this into Eq. (2) and formally solving for the components of the full propagator, we arrive at

$$
\begin{aligned}
T^{ \pm}= & -Z_{1 F}\left(Z_{2} S_{0}^{\mp-1}+Z_{1 F} \Sigma^{\mp}\right)^{-1} \Phi^{ \pm} S^{ \pm}, \\
S^{ \pm-1}= & Z_{2} S_{0}^{ \pm-1}+Z_{1 F} \Sigma^{ \pm} \\
& -Z_{1 F}^{2} \Phi^{\mp}\left(Z_{2} S_{0}^{\mp-1}+Z_{1 F} \Sigma^{\mp}\right)^{-1} \Phi^{ \pm} .
\end{aligned}
$$

Each of these components are, in principle, matrices in flavor, color, and Dirac space. However, since we only consider pairing of quarks with equal flavor, the flavor structure is trivial, and we can consider each flavor separately.

The bare quark propagator is given by

$$
S_{0}^{+}(p)=\left(-i \omega_{p} \gamma_{4}-i \vec{p} \cdot \vec{\gamma}+m_{0}\right)^{-1}
$$

and $S_{0}^{-}(p)=-C S_{0}^{+}(-p)^{T} C$. Here we have introduced the notation $\omega_{p}=p_{4}+i \mu$. Note that we have dropped possible flavor indices for the quark mass $m_{0}$ and the chemical potential $\mu$, which are in general flavor dependent. On the other hand, we exclude "color chemical potentials", i.e., constant gluonic background fields, because it can be shown by the equations of motion that these vanish in the CSL phase.

Defining the gluon momentum by $k=p-q$, the normal self-energy $\Sigma^{+}$and the gap functions $\Phi^{+}$in our truncation are obtained via

$$
\begin{aligned}
\Sigma^{+}(p)=\frac{Z_{2}^{2}}{Z_{1 F}} \pi \int \frac{d^{4} q}{(2 \pi)^{4}} \gamma_{\mu} \lambda_{a} S^{+}(q) \gamma_{\nu} \lambda_{a} \alpha_{s}\left(k^{2}\right) \\
\times\left(\frac{P_{\mu \nu}^{T}}{k^{2}+G(k)}+\frac{P_{\mu \nu}^{L}}{k^{2}+F(k)}\right), \\
\Phi^{+}(p)=-\frac{Z_{2}^{2}}{Z_{1 F}} \pi \int \frac{d^{4} q}{(2 \pi)^{4}} \gamma_{\mu} \lambda_{a}^{T} T^{+}(q) \gamma_{\nu} \lambda_{a} \alpha_{s}\left(k^{2}\right) \\
\times\left(\frac{P_{\mu \nu}^{T}}{k^{2}+G(k)}+\frac{P_{\mu \nu}^{L}}{k^{2}+F(k)}\right) .
\end{aligned}
$$


Furthermore, we have $\Sigma^{-}(p)=-C \Sigma^{+}(-p)^{T} C$ and $\Phi^{-}(p)=\gamma_{4} \Phi^{+}(p)^{\dagger} \gamma_{4}$, which guarantees the action to be real [2, 20]. Together with Eqs. (4) and (5), these expressions form a set of integral equations which have to be solved self-consistently.

$P_{\mu \nu}^{T}$ and $P_{\mu \nu}^{L}$ are the usual projectors in the subspace orthogonal to the gluon momentum $k_{\mu} . P_{\mu \nu}^{T}$ is transverse to the 3 -momentum $\vec{k}$, whereas $P_{\mu \nu}^{L}$ is longitudinal. The functions $G(k)$ and $F(k)$ encode Landau damping and Debye screening of the gluons. In our analysis this is not included self-consistently, but for simplicity $F$ and $G$ are computed using bare quark propagators. The result has similarities with the hard-dense-loop approximation at asymptotically large densities. (For details, see Ref. [15].)

The function $\alpha_{s}\left(k^{2}\right)$, being referred to as the strong running coupling, is the only input in our truncation scheme. Analogous to previous investigations of the $2 \mathrm{SC}$ and CFL phase [15, 17], we employ two different couplings. One of theses running couplings, named $\alpha_{\mathrm{I}}\left(k^{2}\right)$, has been determined in DSE studies of the Yang-Mills sector [21]. It will serve as a lower bound to the coupling strength, since it underestimates chiral symmetry breaking significantly in our vertex approximation. The other running coupling considered is chosen to reproduce lattice QCD results for the quark and gluon propagators in the vacuum for Landau gauge [22] and will be labeled $\alpha_{\mathrm{II}}\left(k^{2}\right)$. In order to vary the renormalization point for this coupling, we exploit the multiplicative renormalizability of the quark DSE.

As stated above, taking into account the medium polarization, Debye screening and Landau damping are included. Both, screening and damping of the interaction, increase with the interaction strength. As a consequence the generated gap functions are relatively insensitive to variations of $\alpha_{s}\left(k^{2}\right)$. Due to this fortunate instance, our approach has quite a predictive power despite the uncertainties in the effective low-energy quark interaction.

The renormalization constants are determined in the chirally broken vacuum. Inserting the above expressions for the normal and anomalous self-energy into the DSE, $Z_{1 F}$ cancels out. We determine the quark wave function renormalization constant $Z_{2}$ and the renormalization constant $Z_{m}$, relating the unrenormalized quark mass $m_{0}\left(\Lambda^{2}\right)$ at a cutoff $\Lambda$ to the renormalized mass $m(\nu)$ via

$$
m_{0}\left(\Lambda^{2}\right)=Z_{m}\left(\nu^{2}, \Lambda^{2}\right) m(\nu),
$$

by requiring

$$
\left.S_{q}^{+}(p)\right|_{p^{2}=\nu^{2}}=-i \not p+m(\nu)
$$

at a renormalization scale $\nu$. The mass dependence of the quark wave function renormalization constant $Z_{2}$ turns out to be negligible in the considered mass ranges. Therefore $Z_{2}$ is determined in the chiral limit.

\section{B. General CSL symmetric ansatz}

As pointed out before, the truncated Dyson-Schwinger equation presented above corresponds to a coupled set of integral equations. In order to find a self-consistent solution, we parameterize the normal and anomalous selfenergies in terms of scalar functions (so-called dressing functions) and determine normal and anomalous propagators via Eqs. (4) and (5). These are then inserted into Eqs. (77) and (8) to recalculate the self-energies, and the whole procedure is iterated until convergence is achieved.

For this strategy we need to ensure that the basis for the parameterization of the self-energies and propagators forms a closed set, i.e., that no further structures are generated during the iteration. To that end, we must allow for the most general parameterization which is consistent with all symmetries that are left unbroken in the considered phase, i.e., in our case, the CSL phase.

In the Introduction we loosely characterized the CSL phase as spin-1 pairing of quarks with equal flavor, where the three spin projections are locked to the three color degrees of freedom. We should now be more precise: In the CSL phase, the total (spin + orbital) angular momentum of the pair is locked to an $S O_{c}(3)$ subgroup of the $S U_{c}(3)$ color symmetry. This means, the CSL ground state remains invariant under transformations of the quark fields, generated by the sum [8]

$$
\vec{G}=\vec{J}+\vec{I},
$$

where $\vec{J}=\vec{S}+\vec{L}, \vec{S}=\frac{i}{2} \gamma_{5} \gamma_{4} \vec{\gamma}$ is the spin operator, $\vec{L}=\vec{r} \times \vec{p}$ is the orbital angular momentum, and $\vec{I}=\left(\lambda_{7},-\lambda_{5}, \lambda_{2}\right)$ is the generator of the above-mentioned $S O_{c}(3)$ subgroup in color space. One can easily verify that the generators $\vec{G}$ satisfy the commutation relations

$$
\left[G_{i}, G_{j}\right]=i \epsilon_{i j k} G_{k},
$$

corresponding to a residual $S U_{J+c}(2)$ symmetry.

For a CSL invariant ground state, all Green functions are also invariant under CSL transformations. Therefore, we require for the normal and anomalous propagators

$$
\begin{aligned}
& S^{+}\left(\vec{p}, p_{4}\right)=U S^{+}\left(\vec{p}, p_{4}\right) \gamma_{4} U^{\dagger} \gamma_{4}, \\
& T^{+}\left(\vec{p}, p_{4}\right)=U T^{+}\left(\vec{p}, p_{4}\right) C^{-1} U^{T} C,
\end{aligned}
$$

where $U \in S U_{J+c}(2)$. In addition, we restrict ourselves to even parity pairing, i.e., we require

$$
\begin{aligned}
& S^{+}\left(\vec{p}, p_{4}\right)=\gamma_{4} S^{+}\left(-\vec{p}, p_{4}\right) \gamma_{4}, \\
& T^{+}\left(\vec{p}, p_{4}\right)=-\gamma_{4} T^{+}\left(-\vec{p}, p_{4}\right) \gamma_{4} .
\end{aligned}
$$

We will now present a self-consistent parameterization under these conditions. Since only quarks with equal flavor are pairing in the CSL phase, the different flavors decouple completely in our truncation. (In fact, the number of flavors only appears in the medium polarization, i.e., as a prefactor in the screening functions $F(k)$ and 
$G(k)$.) As a consequence, we only need to parameterize the color-Dirac structure. Nevertheless, the number of terms which are consistent with our requirements - and therefore have to be considered - is still very large: We find that normal and anomalous self-energies as well as normal and anomalous propagators must be parameterized by 20 independent functions each.

The result can be written in the following form,

$$
\begin{aligned}
& S^{+}(p)=Z_{2} \sum_{i=1}^{5}\left(-i \vec{p} \cdot \vec{\gamma} S_{A, i}^{+}(p)-i \omega_{p} \gamma_{4} S_{C, i}^{+}(p)\right. \\
& \left.+S_{B, i}^{+}(p) \quad-i \gamma_{4} \vec{p} \cdot \vec{\gamma} S_{D, i}^{+}(p)\right) P_{i}, \\
& T^{+}(p)=Z_{2} \sum_{i=1}^{5}\left(i \gamma_{4} \hat{p} \cdot \vec{\gamma} T_{A, i}^{+}(p)+\gamma_{4} T_{B, i}^{+}(p)\right. \\
& +T_{C, i}^{+}(p) \quad+i \hat{p} \cdot \vec{\gamma} T_{D, i}^{+}(p) \quad M_{i}, \\
& \Sigma^{+}(p)=\frac{Z_{2}}{Z_{1 F}} \sum_{i=1}^{5}\left(-i \vec{p} \cdot \vec{\gamma} \Sigma_{A, i}^{+}(p)-i \omega_{p} \gamma_{4} \Sigma_{C, i}^{+}(p)\right. \\
& \left.+\Sigma_{B, i}^{+}(p) \quad-i \gamma_{4} \vec{p} \cdot \vec{\gamma} \Sigma_{D, i}^{+}(p)\right) P_{i}, \\
& \Phi^{+}(p)=\frac{Z_{2}}{Z_{1 F}} \sum_{i=1}^{5}\left(i \gamma_{4} \hat{p} \cdot \vec{\gamma} \phi_{A, i}^{+}(p)+\gamma_{4} \phi_{B, i}^{+}(p)\right. \\
& \left.+\phi_{C, i}^{+}(p) \quad+i \hat{p} \cdot \vec{\gamma} \phi_{D, i}^{+}(p) \quad\right) M_{i},
\end{aligned}
$$

where $P_{i}$ and $M_{i}$ are matrices in color-Dirac space defined by

$$
\begin{aligned}
& \left(P_{1}\right)_{i j}=\delta_{i j} \\
& \left(P_{2}\right)_{i j}=-\gamma_{5}\left(\hat{p} \cdot \vec{I}_{i j}\right) \\
& \left(P_{3}\right)_{i j}=\gamma_{4} \gamma_{5}\left(\vec{\gamma} \cdot \vec{I}_{i j}\right) \\
& \left(P_{4}\right)_{i j}=\hat{p}_{i} \hat{p}_{j} \\
& \left(P_{5}\right)_{i j}=\gamma_{4}\left(\gamma_{i} \hat{p}_{j}+\hat{p}_{i} \gamma_{j}\right)
\end{aligned}
$$

and $M_{i}=\gamma_{5} \gamma_{4} P_{i}$. The indices $i, j$ are indices in color space while the Dirac structure is implicit. As before, $\hat{p}=\vec{p} /|\vec{p}|$.

This ansatz can be derived by considering the largest linear independent basis in color-Dirac space under the $S U_{J+c}(2)$ symmetry. It can furthermore be deduced by coupling two quark fields in three internal $S U(2)$ representations to an overall singlet. Our notation of factorizing the right hand sides of Eq. (15) into four pure Dirac terms and the color-Dirac matrices $P_{i}$ or $M_{i}$ is mainly motivated by the structure of the propagators and self-energies in a normal-conducting medium, which are unit matrices in color space $\left(\equiv P_{1}\right)$ and are customarily parameterized by the same Dirac terms. Also note that the functions $S_{F, i}^{+}(p), T_{F, i}^{+}(p) \Sigma_{F, i}^{+}(p)$, and $\phi_{F, i}^{+}(p)$ are renormalization point independent. Moreover, those terms with $F=A$ or $F=C$ are chirally symmetric, whereas the terms with $F=B$ or $F=D$ break chiral symmetry. We want to emphasize, that the tensor structure is completely determined by the residual symmetry and therefore independent of our truncation.

The defining equation for the anomalous propagator, Eq. (4), is homogeneous. Therefore we are free to choose a global phase for the gap function. In our calculations, we chose this phase, such that

$$
\phi_{F, i}^{+}\left(p_{4},|\vec{p}|\right)=\phi_{F, i}^{+}\left(-p_{4},|\vec{p}|\right)^{*}
$$

and therefore, all scalar gap functions are real for $p_{4}=0$.

Having discussed the most general ansatz for the CSL phase under even-parity pairing, we can map out previous non-self-consistent ansätze by their specific parameterization of the gap functions as given in Eq. (15). For the ansatz used in Ref. [11] the mapping is particularly simple. It is given by

$$
\phi_{C, 3}^{+}=\Delta
$$

and all other scalar gap functions vanish.

A more elaborate ansatz has been chosen in Refs. 2, 8, 10]. In addition, there was also a distinction between different cases of the CSL phase. One of these cases, the so-called "transverse case" maps into our notation by

$$
\begin{gathered}
\phi_{A, 2}^{+}=\phi_{C, 3}^{+}=\frac{1}{2}\left(\phi^{+}+\phi^{-}\right), \\
\phi_{A, 3}^{+}=\phi_{C, 2}^{+}=\frac{i}{2}\left(\phi^{+}-\phi^{-}\right),
\end{gathered}
$$

with $\phi^{ \pm}$being the (anti-)quasiparticle gap functions in the notation of Refs. [2, 10]. In weak-coupling calculations $\phi^{-}$is set to zero, since it does not contribute to $\phi^{+}$ even to subsubleading order [2].

A second ansatz discussed in Refs. [2, 10] is called the "mixed case" and breaks chiral symmetry. For this case the mapping is

$$
\begin{aligned}
\phi_{A, 2}^{+} & =\phi_{C, 3}^{+}=\phi_{B, 2}^{+}=\frac{1}{2}\left(\phi^{+}+\phi^{-}\right), \\
\phi_{A, 3}^{+}=\phi_{C, 2}^{+} & =-\phi_{D, 2}^{+}=\frac{i}{2}\left(\phi^{+}-\phi^{-}\right) .
\end{aligned}
$$

In the weak-coupling limit, where again $\phi^{-}$is set to zero, this ansatz is energetically disfavored compared to the ansatz given by Eq. (19).

\section{RESULTS}

\section{A. The scalar gap functions as functions of the chemical potential}

We begin with the numerical results for the gap functions $\phi_{F, i}^{+}$at the Fermi surface, i.e., at $p_{4}=0$ and $|\vec{p}|=p_{F}$. The Fermi momentum $p_{F}$ corresponds to the 

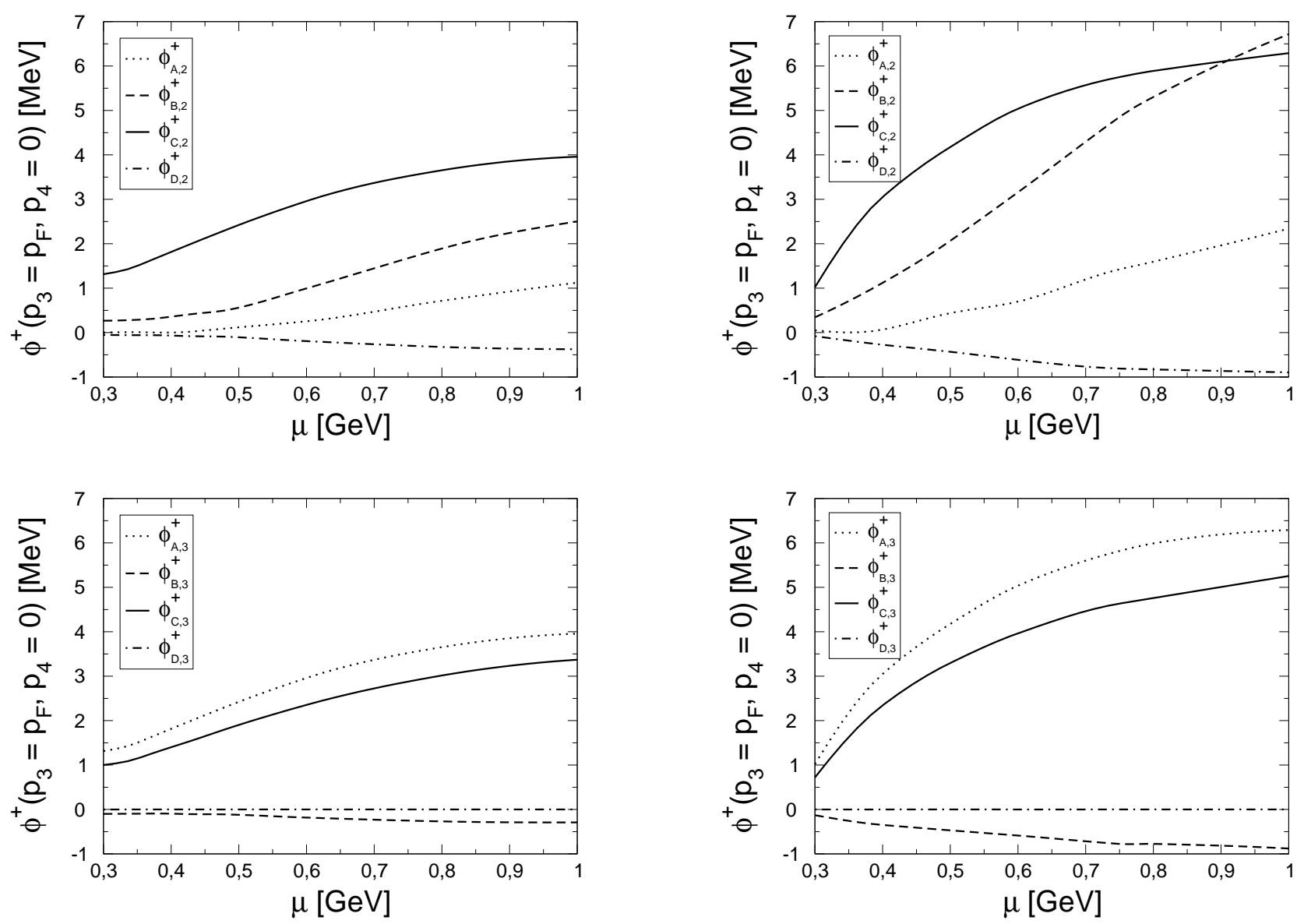

Figure 1: The values of the gap functions $\phi_{F, 2}^{+}$(upper panel) and $\phi_{F, 3}^{+}$(lower panel), $F=A, B, C, D$, at the Fermi surface for quarks with a renormalized mass of $18.4 \mathrm{MeV}$ at the renormalization point $\nu=2 \mathrm{GeV}$, obtained with the strong running coupling $\alpha_{\mathrm{I}}\left(k^{2}\right)$.

momentum where the gap functions have their maxima. ${ }^{1}$ We restrict our discussion to the gap functions $\phi_{F, 2}^{+}$and $\phi_{F, 3}^{+}$, which correspond to the attractive channels. The other gap functions are induced by self-consistency, but their size turns out to be negligible.

Our results as functions of the chemical potential $\mu$ are shown in Fig. 1, which has been obtained with the (weaker) strong running coupling $\alpha_{\mathrm{I}}\left(k^{2}\right)$, and in Fig. 2, which corresponds to $\alpha_{\mathrm{II}}\left(k^{2}\right)$. As one might expect, there is a general tendency that the chiral symmetry conserving

\footnotetext{
${ }^{1}$ In general, the Fermi momenta can be defined via Luttinger's theorem, see Ref. [15]. In the present case, however, the much simpler approximation as being located at the maxima of the gap functions works extremely well. As the gaps are relatively small, the anomalous propagator is strongly peaked at the Fermi surface and, consequently, also the gap function. The error due to this approximation is small compared with the shift of the Fermi momenta due to the normal self-energy.
}

Figure 2: The same as Fig. 1, but for the running coupling $\alpha_{\mathrm{II}}\left(k^{2}\right)$ with a renormalized mass of $18 \mathrm{MeV}$ at the renormalization point $\nu=2 \mathrm{GeV}$.

gap functions, $\phi_{A, i}^{+}$and $\phi_{C, i}^{+}$, are more important than the the symmetry breaking ones, $\phi_{B, i}^{+}$and $\phi_{D, i}^{+}$. One important exception is the function $\phi_{B, 2}^{+}$which can be as big as or even bigger than the symmetry conserving functions. On the other hand, $\phi_{A, 2}^{+}$is relatively small.

If we compare this with previous works, we see that the NJL ansatz, Eq. (18), is a rather poor approximation. But also the "transverse case", Eq. (19), misses the effect of $\phi_{B, 2}^{+}$. Only in the "mixed case", Eq. (20) all important gap functions are taken into account, although not as independent quantities. In fact, it is interesting to note that, within numerical precision, we find that $\phi_{C, 2}^{+}=$ $\phi_{A, 3}^{+}$, in agreement with Eqs. (19) and (20).$^{2}$ The other identities in these equations, however, are not found to hold.

\footnotetext{
2 This seems to indicate that this identity is protected by an underlying symmetry. However, until now, we have not been able to identify it.
} 
The scalar gap functions are of the order of a few $\mathrm{MeV}$. This is about two orders of magnitude larger than one would have expected from extrapolating the weakcoupling results of the CSL phase to small chemical potentials. This remarkable result can be traced back to two features: First, already for spin-0 phases, our approach leads to gap functions which are one order of magnitude larger than the extrapolated weak-coupling results [15]. And second, the suppression of the of the spin- 1 gaps relative to the spin- 0 gap is also about one order of magnitude less than in the weak-coupling limit. E.g., for the "transverse case", the latter is given by a factor of $e^{-9 / 2}$, which is the product of two equal suppression factors of $e^{-9 / 4} \approx 0.1$ related to longitudinal and transverse gluons, respectively [10]. However, when we switch off the longitudinal gluons by hand, we find that their contribution is almost negligible in our approach and, therefore, the suppression relative to the spin-0 result is only about one order of magnitude.

Comparing Fig. 1 with Fig. 2, we find that the different scalar gap functions respond differently to the change of the strong running coupling. Typically, the results differ by a factor of 2 , but the effect is somewhat smaller for $\phi_{C, 2}^{+}, \phi_{A, 3}^{+}$and $\phi_{C, 3}^{+}$, whereas for $\phi_{B, 2}^{+}$it is about a factor of 2.5 at $\mu=1 \mathrm{GeV}$ and even a factor of 4 at $\mu=0.5 \mathrm{GeV}$. This is similar to the quark massfunction in the vacuum, which differs by a factor of 2-3 between the two couplings [21].

\section{B. The influence of the current quark mass}

Since quark masses played an important role in the NJL-model study of Ref. [11], we have also investigated the effect of quark masses on the scalar gap functions. In Figs. 3 and 4 the values of $\phi_{C, 2}^{+}$and $\phi_{B, 2}^{+}$at the Fermi surface are shown as functions of the quark chemical potential $\mu$ for various quark masses. Obviously, the gap function $\phi_{C, 2}^{+}$is almost independent of the quark mass, whereas there is a moderate mass dependence of $\phi_{B, 2}^{+}$. Indeed, recalling that $\phi_{B, i}^{+}$and $\phi_{D, i}^{+}$break chiral symmetry, it was our expectation that these gap functions are induced by nonvanishing quark masses and therefore the greater sensitivity of $\phi_{B, 2}^{+}$to the quark mass appears rather natural. To our surprise, however, $\phi_{B, 2}^{+}$remains finite even for vanishing quark masses. This means, chiral symmetry is spontaneously broken by the diquark condensates. As we will see in the next section, this has important consequences for the quasiparticle excitation spectrum.

The results of Figs. 3 and 4 suggest that the sensitivity of $\phi_{B, 2}^{+}$to the quark mass is more pronounced for the weaker coupling $\alpha_{I}\left(k^{2}\right)$ than for the stronger coupling $\alpha_{\mathrm{II}}\left(k^{2}\right)$. In order to analyze this behavior more systematically, the value of $\phi_{B, 2}^{+}$at the Fermi surface is shown in the upper panel of Fig. 5 as a function of the quark mass for fixed $\mu=400 \mathrm{MeV}$ and both running couplings.
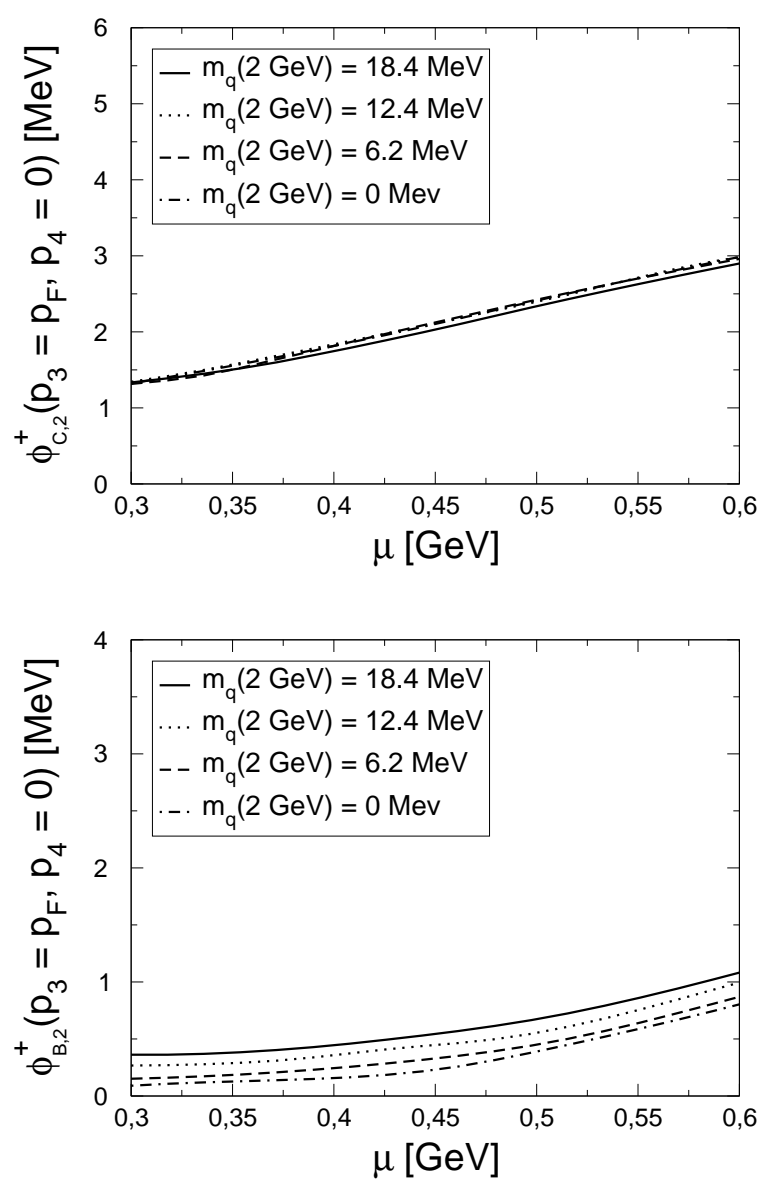

Figure 3: The values of the gap functions $\phi_{C ; 2}^{+}$(upper panel) and $\phi_{B ; 2}^{+}$(lower panel) at the Fermi surface for various quark masses using the strong running coupling $\alpha_{\mathrm{I}}\left(k^{2}\right)$.

For the stronger coupling $\alpha_{\mathrm{II}}\left(k^{2}\right)$, we find that $\phi_{B, 2}^{+}$is almost independent of the quark mass for $m_{q} \lesssim 12 \mathrm{MeV}$ and then becomes more sensitive to the mass (dashed line). This is quite plausible: At low quark masses, the value of $\phi_{B, 2}^{+}$is dominantly determined by the spontaneous symmetry breaking, whereas at higher masses the explicit breaking plays a bigger role.

For the weaker coupling $\alpha_{\mathrm{I}}\left(k^{2}\right)$, on the other hand, the spontaneous symmetry breaking part is much smaller and we find a sizeable mass dependence right from the beginning (solid line). In fact, in this case the mass dependence becomes weaker at larger values of $m_{q}$. This could be due to the fact that at fixed chemical potential the density decreases with the quark mass. Note that $m_{q}$ corresponds to the quark mass at the renormalization point $\nu=2 \mathrm{GeV}$ and the effective masses at the scale of the chemical potential could be considerably larger.

The effect of increasing the chemical potential is very similar to the effect of increasing the coupling strength. This can be seen in the lower panel of Fig. 5 where the value of $\phi_{B, 2}^{+}$at the Fermi surface is shown as a function 

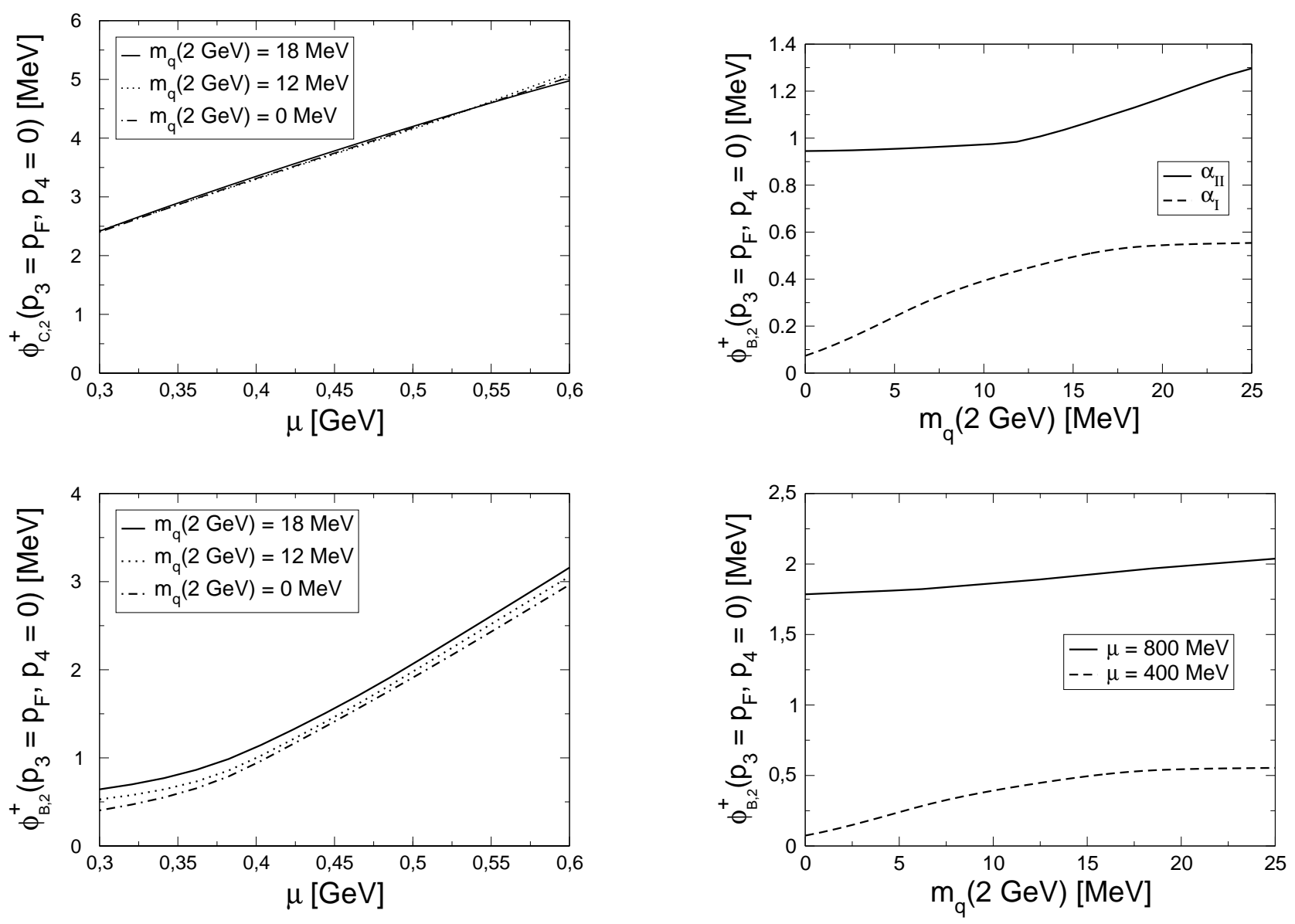

Figure 4: The same as Fig. 3, but using the running coupling $\alpha_{\mathrm{II}}\left(k^{2}\right)$ and slightly different masses.

of the quark mass for the coupling $\alpha_{\mathrm{I}}\left(k^{2}\right)$ and two different chemical potentials. The dashed line corresponds to $\mu=400 \mathrm{MeV}$ and is identical to the dashed line in the upper panel. Increasing the chemical potential to $800 \mathrm{MeV}$ (solid line) we find again that the spontaneous symmetry breaking becomes much stronger and, hence, $\phi_{B, 2}^{+}$becomes less sensitive to the quark mass.

\section{DISPERSION RELATIONS}

\section{A. Discussion}

Since the CSL phase might be relevant for compact star phenomenology, and in particular the question whether there is an ungapped mode in the excitation spectrum, it will be interesting to see how the dispersion relations behave for a self-consistent solution. As mentioned in the Introduction, the dispersion relations for massive quarks in the CSL phase have been investigated in [11] and [14] with contradicting results: The authors of Ref. [14] used a DSE approach in the weak-coupling limit and found

Figure 5: The values of the gap function $\phi_{B ; 2}^{+}$at the Fermi surface as a function of the renormalized quark mass at the renormalization point $\nu=2 \mathrm{GeV}$. Upper panel: Results for $\mu=400 \mathrm{MeV}$ and different running couplings. Lower panel: Results for the running coupling $\alpha_{\mathrm{I}}\left(k^{2}\right)$ and different chemical potentials.

that there is a gapless mode. In the NJL-model investigation of Ref. [11], on the other hand, it was found that there are only gapped modes in the excitation spectrum and that the smallest effective gap is proportional to the constituent quark mass. Since both investigations lack self-consistency, we have the chance to gain more insight into the dispersion relations with our self-consistent ansatz.

To describe the quasiparticle excitation spectrum, in principle we have to extract the spectral functions. This is a relatively difficult task in our approach because one needs to analytically continue the results obtained in Euclidean space into Minkowski space. As demonstrated in Ref. 16] for the case of spin-0 color superconductors, this problem can be solved by applying the maximum entropy method (MEM) 23].

In the following, however, we will pursue a simplified analysis, aiming at a more qualitative discussion. To that end, we concentrate on the dispersion relations $\varepsilon(|\vec{p}|)$ that 
are given as the solutions of the algebraic equation

$$
\left.\operatorname{Det}\left[\mathcal{S}^{-1}\left(\vec{p}, p_{4}\right)\right]\right|_{i p_{4}=\varepsilon(\vec{p})}=0 .
$$

Since our calculations are performed in Euclidean space, we can not access the roots of this equation. Therefore we apply the following approximations: First, we neglect the normal self-energy of the quarks and second, we replace the scalar gap functions in $\Phi^{ \pm}(p)$ by their values at the Fermi surface.

Despite these simplifications, the resulting dispersion relations are extremely long expressions and difficult to handle. Therefore we have searched for the solutions numerically. The results will be presented in the next subsection.

However, to get a deeper understanding of the underlying mechanisms, it is instructive to perform further approximations which allow us to derive analytical expressions for the dispersion relations. In particular, we wish to explore the significance of the gap function $\phi_{B, 2}^{+}$, which we found to be non-negligible in the self-consistent solutions discussed in section III Led by these results, we consider a simple ansatz with

$$
\phi_{A, 3}^{+}=\phi_{C, 2}^{+} \equiv \phi_{C}, \quad \phi_{B, 2}^{+} \equiv \phi_{B},
$$

and neglect all other gap structures. The resulting dispersion relations ${ }^{3}$ are

$$
\varepsilon(|\vec{p}|)= \pm \sqrt{(\mu \pm|\vec{p}|)^{2}+\phi_{B}^{2}}
$$

and

$$
\varepsilon(|\vec{p}|)= \pm \sqrt{(\mu \pm|\vec{p}|)^{2}+\frac{1}{4}\left(\phi_{B} \pm \sqrt{\phi_{B}^{2}+8 \phi_{C}^{2}}\right)^{2}} .
$$

Hence, the simultaneous presence of the gap $\phi_{B}^{+}$, representing opposite chirality pairing, and the gap $\phi_{C}^{+}$, representing equal chirality pairing, implies that all modes are gapped. This is similar to Ref. [10], where the spectrum was found to be fully gapped in the "mixed case", Eq. (20), but not in the "transverse case", Eq. (19).

\section{B. Numerical results}

After these simplified considerations, we now return to the numerical solutions of Eq. (21), using the more complete, although still approximate, expression for the inverse propagator. Since $\phi_{B, 2}^{+} \neq 0$ in the self-consistent solution of the Dyson-Schwinger equations, we expect that all quasiparticle modes will be gapped.

The resulting dispersion relations at $\mu=500 \mathrm{MeV}$ for massive quarks with a renormalized mass of

\footnotetext{
${ }^{3}$ Note, that in this simplified case $p_{F}=\mu$ as we neglect normal self-energy contributions and quark masses.
}

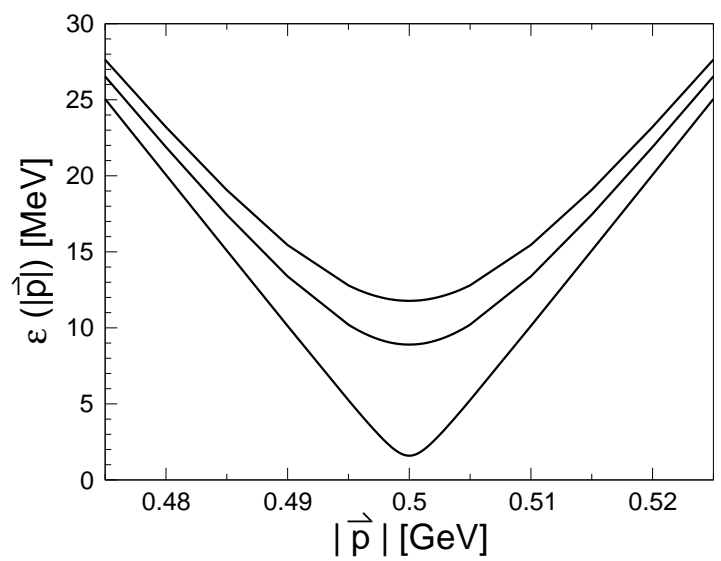

Figure 6: The quasiparticle dispersion relations for quarks with a renormalized mass of $18 \mathrm{MeV}$ at the renormalization point $\nu=2 \mathrm{GeV}$ obtained with the strong running coupling $\alpha_{\mathrm{II}}\left(k^{2}\right)$ at $\mu=500 \mathrm{MeV}$.
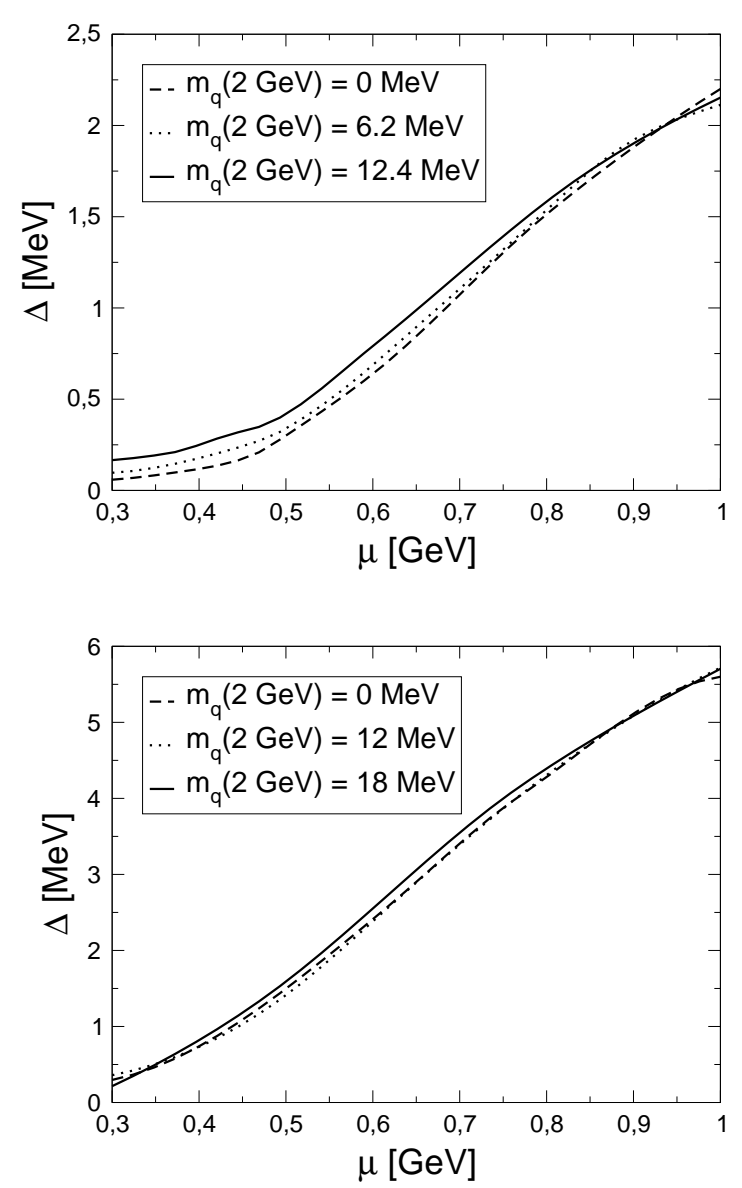

Figure 7: The smallest quasiparticle energy gaps for massive quarks obtained with the running coupling $\alpha_{\mathrm{I}}\left(k^{2}\right)$ (top) and $\alpha_{\mathrm{II}}\left(k^{2}\right)$ (bottom). 
$m_{q}(2 \mathrm{GeV})=18 \mathrm{MeV}$ are shown in Fig. 6. As expected, we observe only gapped modes in the excitation spectrum. Of special interest for compact star cooling phenomenology is the behavior of the size of the smallest effective gap as a function of chemical potential. This is shown in Fig. 7 for the effective strong running couplings $\alpha_{\mathrm{I}}\left(k^{2}\right)$ and $\alpha_{\mathrm{II}}\left(k^{2}\right)$ and for various quark masses. Since we find $\phi_{B, 2}^{+}$to be nonvanishing in the chiral limit, corresponding to a spontaneous symmetry breaking, there are only gapped modes in the excitation spectrum, even for vanishing quark mass. In addition we observe that the dependence of the effective gap on the quark masses is weak, particularly for higher values of the chemical potential. Although the value of the smallest gap is sensitive to the coupling, we can conclude, that the gapfunction is monotonically increasing with the chemical potentials and is of the order of a few $100 \mathrm{keV}$ in the astrophysically relevant regime.

\section{SUMMARY AND CONCLUSIONS}

We have studied the quark propagator in the colorsuperconducting CSL phase at zero temperature in a self-consistent Dyson-Schwinger approach. Our main objective has been the clarification of contradicting predictions by previous investigations, especially concerning the phenomenologically interesting size of the smallest excitation gap. To this end, we have considered the most general ansatz for the quark propagator allowed by the CSL symmetry pattern. For comparison, we have outlined the truncated ansätze of previous investigations and how they are parameterized in our basis. In our numerical exploration, we found that the CSL phase exhibits a color-spin structure that is close to the color-spin structure used in weak-coupling calculations [10]. As a remarkable difference, chiral symmetry breaking terms are additionally dynamically generated, leading to a spontaneous symmetry breakdown. As a result, in contrast to [14], the size of the smallest excitation gap is nonvanishing and triggered by a chiral symmetry breaking diquark condensate. Therefore, the dynamical mass generation, as elaborated in [11], is not necessary for obtaining a nonvanishing smallest excitation gap. Although the value of the smallest gap is sensitive to the coupling, we can conclude, that the gapfunction is increasing monotonically with the chemical potentials and is of the order of a few $100 \mathrm{keV}$ in the astrophysically relevant regime. This seems to be in conflict with the analysis of Ref. [12] where it was concluded that the smallest gap should decrease with the chemical potential in order to describe the neutron star cooling data [12]. However, before drawing strong conclusions, a more careful analysis is certainly required, where both specific heat and neutrino emissivities, are derived within our approach.

\section{ACKNOWLEDGMENT}

We thank R. Alkofer, D. Rischke, A. Schmitt and I. Shovkovy for helpful discussions.

This work has been supported in part by the Helmholtz Association (Virtual Theory Institute VH-VI-041) and by the BMBF under grant number 06DA916.
[1] K. Rajagopal and F. Wilczek, hep-ph/0011333, M. Alford, Ann. Rev. Nucl. Part. Sci. 51, 131 (2001); T. Schäfer, hep-ph/0304281 M. Buballa, Phys. Rep. 407, 205 (2005); H.-C. Ren, hep-ph/0404074; M. Huang, Int. J. Mod. Phys. E 14, 675 (2005); I. A. Shovkovy, Found. Phys. 35, 1309 (2005).

[2] D. H. Rischke, Prog. Part. Nucl. Phys. 52, 197 (2004).

[3] I. A. Shovkovy and L. C. R. Wijewardhana, Phys. Lett. B 470, 189 (1999); T. Schäfer, Nucl. Phys. B575, 269 (2000).

[4] M. G. Alford, K. Rajagopal, and F. Wilczek, Nucl. Phys. B537, 443 (1999).

[5] M. Alford and K. Rajagopal, JHEP 0206, 031 (2002).

[6] S. B. Rüster, V. Werth, M. Buballa, I. A. Shovkovy and D. H. Rischke, Phys. Rev. D 72, 034004 (2005).

[7] H. Abuki and T. Kunihiro, Nucl. Phys. A 768, 118 (2006).

[8] T. Schäfer, Phys. Rev. D 62 (2000) 094007.

[9] M. G. Alford, J. A. Bowers, J. M. Cheyne and G. A. Cowan, Phys. Rev. D 67 (2003) 054018.

[10] A. Schmitt, Phys. Rev. D 71 (2005) 054016.

[11] D. N. Aguilera, D. Blaschke, M. Buballa and V. L. Yudichev, Phys. Rev. D 72, 034008 (2005).

[12] H. Grigorian, D. Blaschke and D. Voskresensky, Phys.
Rev. C 71, 045801 (2005).

[13] D. N. Aguilera, D. Blaschke, H. Grigorian and N. N. Scoccola, Phys. Rev. D 74, 114005 (2006).

[14] A. Schmitt, I. A. Shovkovy and Q. Wang, Phys. Rev. D 73, 034012 (2006).

[15] D. Nickel, J. Wambach and R. Alkofer, Phys. Rev. D 73, 114028 (2006).

[16] D. Nickel, Annals Phys. in print, arXiv:hep-ph/0607224

[17] D. Nickel, R. Alkofer and J. Wambach, arXiv:hep-ph/0609198

[18] R. Alkofer and L. von Smekal, Phys. Rept. 353, 281 (2001); C. S. Fischer, J. Phys. G 32, R253 (2006).

[19] Q. Wang and D. H. Rischke, Phys. Rev. D 65, 054005 (2002).

[20] D. Bailin and A. Love, Phys. Rept. 107, 325 (1984).

[21] C. S. Fischer and R. Alkofer, Phys. Rev. D 67, 094020 (2003).

[22] M. S. Bhagwat, M. A. Pichowsky, C. D. Roberts and P. C. Tandy, Phys. Rev. C 68, 015203 (2003).

[23] M. Jarrell and J. E. Gubernatis, Phys. Rept. 269, 133 (1996); M. Asakawa, T. Hatsuda and Y. Nakahara, Prog. Part. Nucl. Phys. 46, 459 (2001). 